\title{
Oncoplasty: how to start and experience from Yogyakarta
}

\author{
Herjuna Hardiyanto* \\ Division of Surgical Oncology, Department of Surgery Dr.Sardjito Hospital / \\ Faculty of Medicine Universitas Gadjah Mada Yogyakarta, Indonesia
}

DOI: http://dx.doi.org/10.19106/JMedScieSup0048042016013

\section{ABSTRACT}

Reconstruction surgery after tumor removal is increasingly essential due to the changes in patients' expectations and demands. With the advance of early screening and detection, our ability to diagnose early stages of cancer is significantly improved. Reconstruction is mostly suitable in patients with earlystage cancers. In addition, there is a growing ability to perform immediate reconstruction in selected patients, utilizing single-time operation involving oncological and aesthetic procedures with considerably excellent result. With the implementation of full coverage insurance by BPJS, most oncological surgery, including breast cancer surgery, will be performed by general surgeon in regency hospital. Reconstruction in selected patients is usually performed as delayed procedure by plastic surgeon. With the advance in surgical oncology training, breast surgery should be performed by surgical oncologist with specific oncoplasty training that offers immediate reconstruction with both extensive removal tumor and reconstruction. Therefore, training in oncoplasty is very important for surgical oncologist. In Yogyakarta, we performed reconstruction after surgery of breast tumor, thyroid cancer, radical neck dissection, and facial basal cell carcinoma with relatively excellent results. Further training and innovation are required to improve this early stage of oncoplasty practice in Yogyakarta.

Keywords: oncoplasty, breast, thyroid, reconstruction 